Tau-specific antibodies contribute to the preservation of cognitive function.

\title{
Tau immunotherapy targets transcellular propagation
}

Extracellular deposition of amyloid- $\beta$ and intracellular aggregates of tau are pathological hallmarks of Alzheimer's disease. To date, most attempts at developing immunotherapies to treat Alzheimer's disease have focused on targeting amyloid- $\beta$, but a study published in Neuron suggests that antibodies against extracellular tau that block the uptake of seeding competent forms of the protein into cells could be a promising new treatment strategy.

Tau aggregates are observed in a number of neurodegenerative diseases (referred to as tauopathies) and lead to the formation of neurofibrillary tangles that are associated with synaptic dysfunction and cognitive decline. Previous studies have suggested that tau fibrils can be released into the extracellular space and induce aggregation of tau in recipient cells through a process referred to as seeding. On the one hand, this promotes propagation of the pathology but on the other hand it offers an opportunity to target extracellular tau and disrupt the process.

Yanamandra et al. used a cellbased biosensor assay to screen for tau-specific monoclonal antibodies that block tau aggregation induced by the addition of brain lysates from transgenic mice expressing human P301S tau - a mutated form of tau that causes inherited frontotemporal dementia. They selected three effective antibodies with different potencies (HJ8.5, HJ9.3 and HJ9.4) and confirmed their ability to block the seeding activity of P301S tau aggregates in HEK293 cells using a fluorescence resonance energy transfer (FRET) assay. As the antibodies were not detected inside the cells, the authors suggest that their mechanism of action could involve blocking the cellular uptake of seeding competent forms of tau.

When they infused each of the antibodies into the lateral ventricle of 6-month-old P301S mice (which already show signs of tau pathology) for 3 months, they found a significant reduction in phosphorylated tau and insoluble tau, as well as reduced microglial activation in multiple regions of the brain compared to controls. Brain lysates from mice treated with HJ8.5 or HJ9.3 had markedly less seeding activity on HEK293 cells than lysates from controls, which indicates that the reduction of tau aggregation correlates with a reduction in seeding activity. Interestingly, treatment of P301S mice with these antibodies rescued the deficits in associative learning that are present at 9 months of age. Two days after a fear conditioning test, the treated mice (particularly those treated with HJ8.5) showed greater levels of freezing behaviour compared to controls, which suggests that the tau-specific antibodies contribute to the preservation of cognitive function.

Together, these findings highlight the role of extracellular tau in disease pathogenesis and its potential as a target for immunotherapy.

Monica Hoyos Flight

ORIGINAL RESEARCH PAPER Yanamandra, K. et al. Anti-tau antibodies that block tau aggregate seeding in vitro markedly decrease pathology and improve cognition in vivo. Neuron $\mathbf{8 0}, 402-414$ (2013)

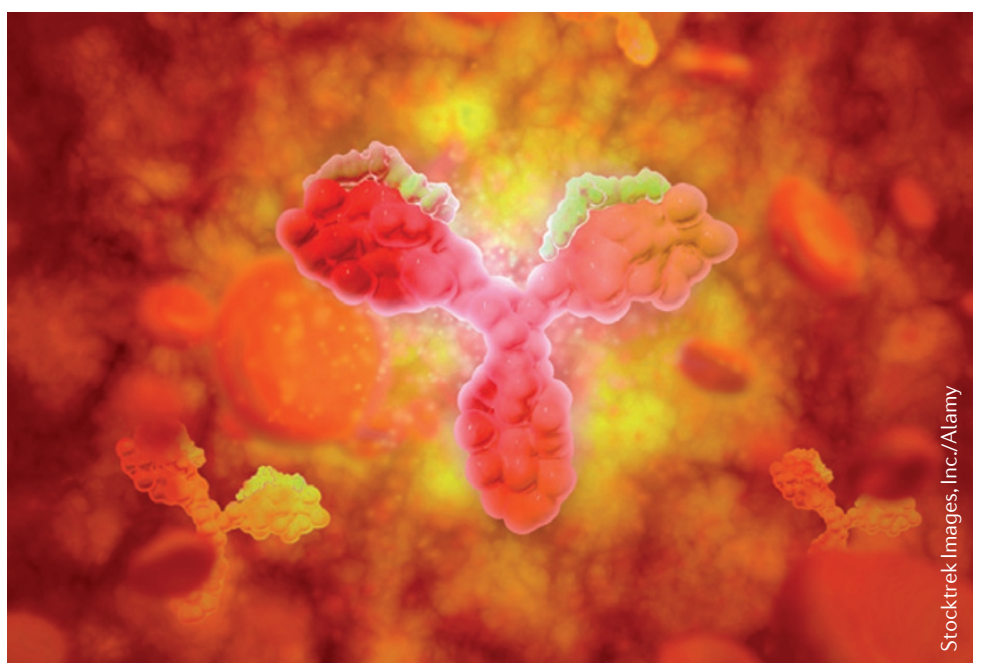

